\title{
Eletromagnetic Survey for Measurement of the Concentration of Aqueous Substances
}

\author{
André Soffiatti and Sandro Gonçalves da Silva
}

\begin{abstract}
This paper shown a analysis of concentration of aqueous substances through of variation of eletric permissivity. In order to investigate this variation of relative permittivity of a medium, we used a pair of microstrip antenna positioned at a distance of $120 \mathrm{~mm}$ and a spectrum analyzer. The first substance analysed was Vinegar (Acetic acid - $\mathrm{CH3COOH)}$ and, thus obtain a pattern to control the variation of the intrinsic characteristics of this substance. This research aims to develop substances control sensors.
\end{abstract}

\section{INTRODUCTION}

This type of testing is essential for a final evaluation before potting these substances and thus accrue control of product quality.

The microstrip antennas are especially attractive because of its low cost, low profile and easy integration with other circuit elements [1]-[3]. In general, they consist of a metallic radiating element, called patch, which can be fed on several ways. The microstrip line is one of the options, but the configuration is widely used in microwave. The patch may have various geometric configurations; in most cases, the loads have input impedances of $75 \Omega$ or $50 \Omega$.

In this sense, the radiation pattern is related to the directivity of the antenna, where one of them is interested in seeing the effect on the incidence of the wave at the frequency generated by the other in the aqueous solution. After the reflection, this wave will focus on the Rx antenna. Observing, thereby, the scattering parameters are S11 and S21.

\section{DEVELOPMENT}

The study investigates the possibility of determining variations in substance monitored for purposes of quality control without using chemicals, providing a check on the process continued in the final stages of analysis and packaging of these substances.

The evaluation process followed a simplified model of analysis of variance of the electric permittivity of the material studied, considered the effects of variation of the resonance frequency of changes to the substance under evaluation.

The geometry of antenna chosen for the analysis was of rectangular shape with insert feed for effects to resonance and impedance matching. The HFSS software was used to design this antenna.

\footnotetext{
Andre Soffiatti, Department of Electrical Engineering and, Sandro Gonçalves da Silva, Department of Communication Engineering, Federal University of Rio Grande do Norte, Natal-RN, Brazil, E-mails: soffiatti.andre@gmail.com, sandro@ct.ufrn.br
}

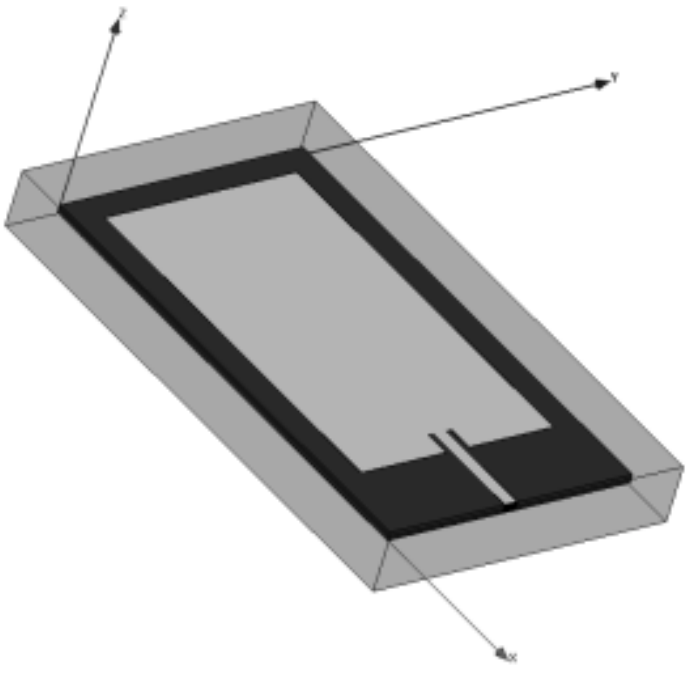

Figure 1. Design of the Antenna

The type of solution used was appropriate for this kind of study because it is colorless and, structurally, the simplest Carboxylic acid.

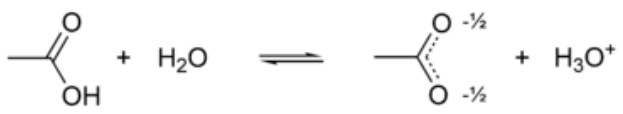

Figure 2. Chemical Reaction between the Acid and Water

Measuring system was composed of a vector network analyzer, the second antenna pattern shown in Figure 1 and an aqueous $\mathrm{C} 2 \mathrm{H} 4 \mathrm{O} 2$ with three levels of concentration that initially contained $1 \%, 8 \%$ and $16 \% \mathrm{C} 2 \mathrm{H} 4 \mathrm{O} 2$. Those were diluted in $100 \mathrm{ml}$ of water. The parser will generate an electromagnetic wave sweeping this case, a frequency range of $500 \mathrm{MHz}$ to $4 \mathrm{GHz}$. This seal wave is transmitted to the antenna through a coaxial cable, thus creating field lines, and the dielectric permittivity is $\varepsilon=4.4$. So, here we have the parameter of S11. For the S21 parameter, the wave will undergo reflection / refraction according to Snell's Law. The incidence of the electromagnetic wave from the two antennas directed identifies the aqueous solution through a change in the behavior of the attenuation network analyzer, and decibel distinguishes each concentration used. 


\section{FIGURES AND TABLES}

TABLE I. PERCENTAGE CONCENTRATION VERSUS ATTENUATION.

\begin{tabular}{|c|c|c|c|}
\hline Concentration & \multicolumn{3}{|c|}{$\begin{array}{c}\text { Aqueous C2H4O2 Solution } \\
\text { Concentration }\end{array}$} \\
\hline & $\begin{array}{c}\text { Milliliter } \\
\mathrm{ml}\end{array}$ & $\begin{array}{c}\text { Percentage } \\
\%\end{array}$ & $\begin{array}{c}\text { Attenuation } \\
\mathrm{dB}\end{array}$ \\
\hline $\mathrm{C} 2 \mathrm{H} 4 \mathrm{O} 2$ & 100 & 1 & $-35,6$ \\
\hline $\mathrm{C} 2 \mathrm{H} 4 \mathrm{O} 2$ & 100 & 8 & $-36,5$ \\
\hline C2H4O2 & 100 & 16 & $-37,1$ \\
\hline
\end{tabular}

In the results below show graphs of measurements to analyze the variation in the percentage of the substances and therefore the sensitivity adjustment can be determined by generating the reference table of the concentration by means of attenuation.

The graph below, we can clearly notice the difference in decibels between the three types of concentration regarding the solution of $\mathrm{C} 2 \mathrm{H} 4 \mathrm{O} 2$ searched.

Initially the $\mathrm{X}$ axis the frequency varies from $2.22 \mathrm{GHz}$ to $2.23 \mathrm{GHz}$. It is considered to reading the graphic $2.222 \mathrm{GHz}$.

On the Y-axis glimpse attenuation is in decibels. The colors in the subsequent table are the following: Blue which represents the vacuum; Red, $\mathrm{C} 2 \mathrm{H} 4 \mathrm{O} 2$ with the percentage of $1 \%$; Green, $\mathrm{C} 2 \mathrm{H} 4 \mathrm{O} 2$ with the percentage of $8 \%$; Yellow, $\mathrm{C} 2 \mathrm{H} 4 \mathrm{O} 2$ with percentage of $16 \%$. [7]-[8].

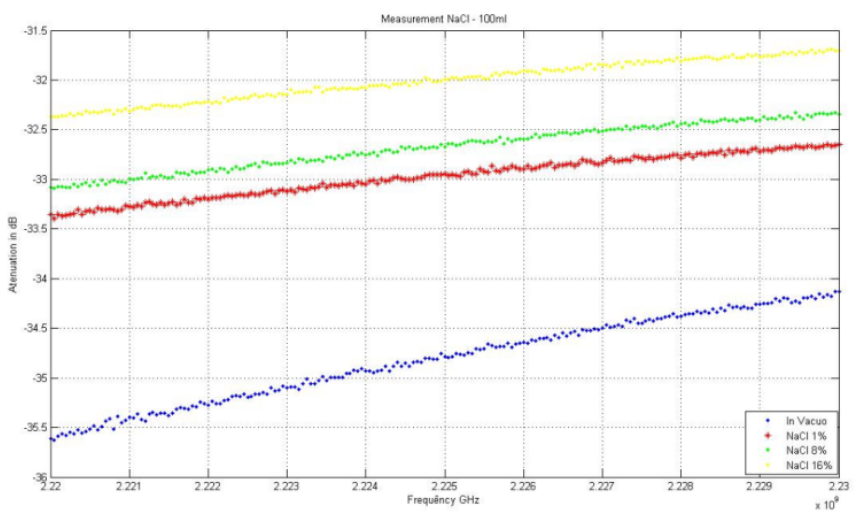

Figure 3. Percentage of the concentration versus Attenuation

\section{EQUATIONS AND THEOREMS}

For the antenna design was used equations below:

Equation 1 is to calculate the width "W" of the antenna. These equations are used to obtain a typical behavior of a frequency of an operation. This paper presented a frequency of $2.4 \mathrm{GHz}$

$$
W=\frac{v_{0}}{2 f r} \sqrt{\frac{2}{\varepsilon_{r}+1}}
$$

Substituting the values of frequency $=2.4 \mathrm{GHz}, \varepsilon_{r}=4.4$ and

$v 0=3 \cdot 10^{8} \mathrm{~m} / \mathrm{s}$.
Velocity of propagation in dielectric waveguide. So we have this: $\mathrm{W}=38 \mathrm{~mm}$, where $\mathrm{C}$ is the velocity of the light in free space.

Calculation to $\varepsilon_{\text {ref }}$ :

$$
\varepsilon_{r e f}=\frac{\varepsilon_{r+1}}{2}+\frac{\varepsilon_{r-1}}{2}\left(1+12 \frac{h}{w}\right)=0,817
$$

Calculation $\mathrm{L}$ we need $\Delta L$ :

$$
0,412 \frac{\left(\varepsilon_{r e f}+0,3\right)\left(\frac{w}{h}+0,264\right)}{\left(\varepsilon_{r e f}-0,258\right)\left(\frac{w}{h}+0,8\right)}=0,831 \mathrm{~mm}
$$

Thus we have L:

$$
L=\frac{1}{2 f r \sqrt{\varepsilon_{r e f}} \sqrt{\mu_{0} \varepsilon_{0}}}-2 \Delta L=67 \mathrm{~mm}
$$

Having found the values fix, they're replaced for the appropriate values of antenna size for it to work in $2.4 \mathrm{GHz}$.

Equation (4) calculates the length "L" of the antenna. These equations are used to obtain a typical behavior for an operating frequency in this $2.4 \mathrm{GHz}$.

\section{CONCLUSIONS}

From the data shown above it is observed that there was a variation $\mathrm{S} 21$ of the setup shown from the substance concentration $\mathrm{C} 2 \mathrm{H} 4 \mathrm{O} 2$ determining the distinction of the solution through a physical procedure with incidence of an electromagnetic wave it is found that using a physical method can replace the usual chemical methods.

\section{REFERENCES}

[1] C.A Balanis, Analysis and Design, 2nd ed., Wiley, New York, 1997.

[2] J. X. Xiao, M.F. Wang, and G.J. Li, A ring monopole antenna for UWB application, Microwave Opt Technol Lett 52 (2010), 179-182.

[3] WEAR, K.A. The Effects of Frequency-dependent Attenuation and Dispersion on Sound Speed Measurements: Application in Human Trabecular Bone. IEEE Transaction on Ultrasonics, Ferroelectrics and Frequency Control, 47(1):265-273, 2000.

[4] V. G. Veselago, "The eletrodynamics of substances with simultaneously negative values of and _, Sov. Phys. Uspekhi, vol. 10, pp. 509-514, Jan.-Feb. 1968.

[5] D. R. Smith, W. J. Padilla, D. C. Vier, S. C. Nemat-Nasser, and S. Schultz, "Composite medium with simultaneously negative permeability and permittivity," Phys. Rev. Lett., vol. 84, pp. 41844187, May 2000.

[6] T. Itoh and C. Caloz, Electromagnetic Metamaterials: Transmission Line Theory and Microwave Applications. Hoboken, NJ: Wiley, 2006, pp. 4-9.

[7] K. Buell, H. Mosallaei, and K. Sarabandi, "A substrate for small patch antennas providing tunable miniaturization factors," IEEE Trans.Microw. Theory Tech., vol. 54, no. 1, pp. 135-146, Jan. 2006.

[8] Z. Li, K. Aydin, and E. Ozbay, "Determination of the effective constitutive parameters of bianisotropic metamaterials from the reflection and transmission coefficients," Phys. Rev. E, vol. 79, pp. 026610-1-026610-7, Feb. 2009. 
H. Mosallaei and K. Sarabandi, "Design and modeling of patch antenna printed on magneto-dielectric embedded-circuits metasubstrate," IEEE Trans. Antennas Propag., vol. 55, no. 1, pp. 4552, Jan. 2007.

C. F. L. Vasconcelos, S. G. Silva, M. R. M. L. Albuquerque, J. R. S.

Oliveira, and A. G. d'Assunção, "Annular ring microstrip antennas for millimeter wave applications," Int. J. Infrared Millim. Waves, vol. 28 ,

pp. 821-829, Jul. 2007.

R. M. Nelson, D. A. Rogers, and A. G. d'Assunção, "Resonant frequency of a rectangular microstrip patch on several uniaxial layers,"

IEEE Trans. Antennas Propag., vol. 38, no. 7, pp. 973-981, Jul. 1990.

[9] H. How, T. M. Fang, and C. Vittoria, "Intrinsic modes of radiation in ferrite patch antennas," IEEE Trans. Microw. Theory Tech., vol. 42, no. 6, pp. 988-994, Jun. 1994.

D. M. Pozar, "Radiation and scattering characteristics of microstrip antennas on normally biased ferrite substrates," IEEE Trans. Antennas Propag., vol. 40, no. 9, pp. 1084-1092, Sep. 1992. 